\title{
MEMS Gyro Random Error On-line Modeling Based on Recursive Least-Squares Method of System Identification
}

\author{
Jiawei Wang ${ }^{1,2, a}$, Keyu Qi ${ }^{2}$, Kai Shi ${ }^{2}$, Ke Liang $^{2}$ and Jie Yan ${ }^{1}$ \\ ${ }^{1}$ Northwestern Polytechnical University, 710072 Xi'an China \\ ${ }^{2}$ Science and Technology on Electromechanical Dynamic Control Laboratory, 710065 Xi'an China \\ awangjiaw0126@163.com
}

Keywords: Gyro Random Error, On-line Modeling, System Identification, Recursive Least-Squares Method

\begin{abstract}
To develop the guided projectile with higher hit precision, promotes gyro's performance has immensely affection on trajectory measurement. According to the difficulty in method of MEMS angular rate gyro random error on-line modeling, this paper proposes a new algorithm of recursive least-squares method that used in the system identification processing. With this recursive least-squares method, a few known gyro output data are used to roughly estimate system parameters, then newly obtained data are added to the measurement formula using the recursive algorithm in order to make estimated parameters approach to its truth-value. This study indicates that the residual error between outputs of gyro random error model and outputs of real gyro highly approximates white noise, which means the error model accurately describes statistical features of gyro random error and the recursive algorithm realizes on-line modeling.
\end{abstract}

\section{Introduction}

Modern battlefield's missions require lower collateral damage and higher hit precision. To meet this requirement, existing gun-lunched artillery projectile should possess high precise guidance and navigation abilities, which mean better performance of inertial navigation kit is needed. Known to all, satellite positioning and angular rate gyro are widely used in navigation field to measure attitude of guided projectile, thus gyro's own precision has immensely influence on guided weapon [1].

Normally there are two ways to solve the problem [2]: first one is to enhance the process technology which will cost enormously, another way is to build precise gyro output model for simulating the actual fact as possible which will be more reasonable. Gyro's measurement error mainly contains determinate error and random error, for determinate error gyro's I/O data always be used to form multiple-term formula system, then polynomial coefficient need to be solved and finally system parameters are identified. For gyro random error, PSD method and Allen variance calculations are widely used, however two well-known ways above are all off-line modeling method that not fit for guided projectile on-line modeling, because of the high real-time requirement [3].

According to the difficulty of gyro random error on-line modeling, this paper proposes a new algorithm of recursive least-squares method that used in the system identification processing. This study indicates that the residual error between outputs of gyro random error model and outputs of real gyro highly approximates white noise, which means the error model accurately describes statistical features of gyro random error and the recursive algorithm realizes on-line modeling.

\section{Research Foundation}

This section introduces information of the previous methods to deal with gyro output model, form of ARMA system model and least-squares method to estimate the system parameters.

A. Gyro Random Error Analysis Based on Time-Series Model [4]

For linear system, discrete time series models are well suited for automatic digital identification. One such model which is attractive for this application because it is a minimum parameter model is 
the Autoregressive Moving Average model (ARMA). The basic ARMA model for a single input and single output (SISO) system has the form

$$
x_{t}+\phi_{1} x_{t-1}+\phi_{2} x_{t-2}+\cdots+\phi_{p} x_{t-p}=u_{t}+\theta_{1} u_{t-1}+\theta_{2} u_{t-2}+\cdots \theta_{q} u_{t-q}
$$

Where $x_{t}(t=1,2,3 \cdots)$ is the system response at the $k$ th discrete time, $u_{t}$ is the system input at the $k$ th discrete time, $p$ and $q$ are the order or size of the model, the $a$ 's and the $b$ 's are the model's parameters that should be identified. Now structure of ARMA model is made firstly. Secondly, AIC criteria is chosen to be the model order selection criteria and least-squares system identification method is needed. Finally, examines the residual error between model outputs and real gyro outputs to check if it highly closes to white noise [5,6]. Here are the steps of time-series modeling with following figure.

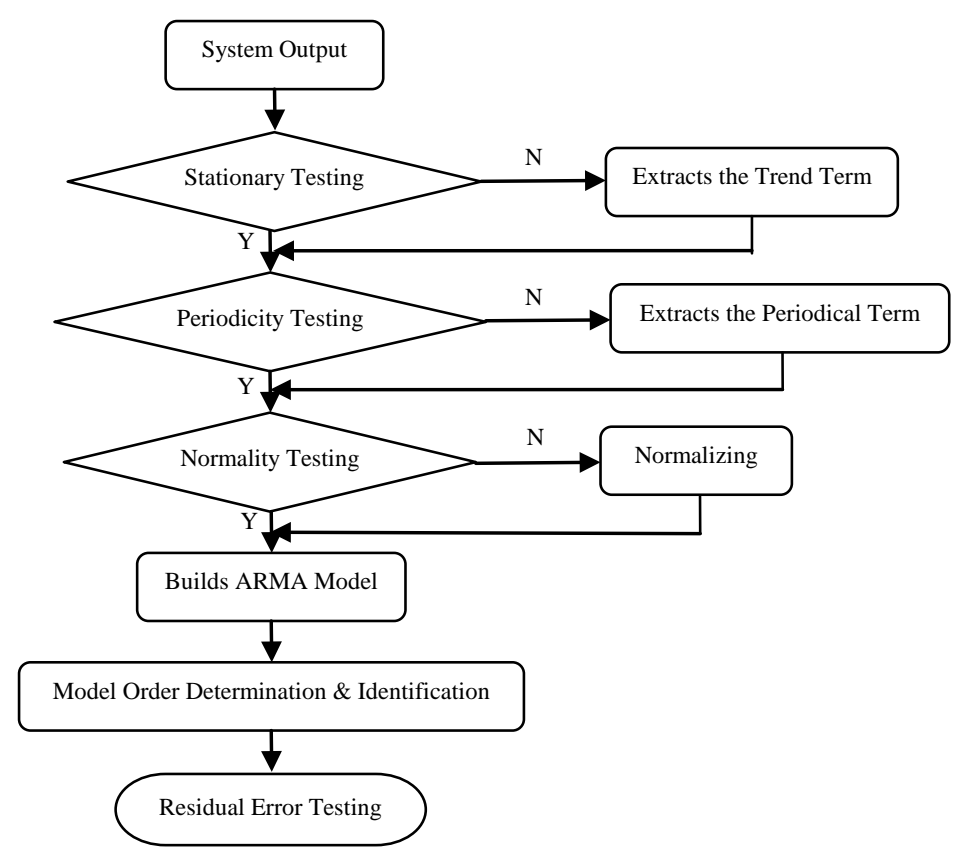

Figure 1. Key Steps of Time-Series Modeling

B. Least-Squares System Identification [7]

Hypothetically, the system's difference equation models are given as follows.

$$
\left\{\begin{array}{c}
x(k)+a_{1} x(k-1)+\cdots+a_{n} x(k-n)=b_{0} u(k)+\cdots b_{n} u(k-n) \\
y(k)=x(k)+n(k)
\end{array}\right.
$$

System output formula can be easily written as following equations.

$$
\mathrm{Y}_{N \times 1}=\Phi_{N \times(2 n+1)} \theta_{(2 n+1) \times 1}+\xi_{N \times 1}
$$

Where $\Phi_{N \times(2 n+1)}$ is measurement matrix, $\theta_{(2 n+1) \times 1}$ is system parameters to be estimate, $\xi_{N \times 1}$ is residual error, and $\mathrm{Y}_{N \times 1}$ is system output.

System parameters identifies with least-squares method is given below.

$$
\widehat{\theta}=\left(\Phi^{T} \Phi\right)^{-1} \Phi^{T} Y
$$

Learning from above equations, basic least-squares method is a kind of off-line system identification utilizing already known I/O data, which is not fit for guided projectile because of lacking real-time ability.

\section{RLS Method Solves On-line Modeling}

This section gives detail method and key steps of brand new recursive least-squares to solve gyro random error on-line modeling that using a kind of recursive operator. 
The core concept is that using least-squares method to roughly estimate the approximation of system parameters from limited known data firstly, then utilizing newly data with recursive calculating algorithm to approach its real value gradually. Recursive steps are given below.

Step 1. Hypothetically, the length of known I/O data is $N$, roughly estimation is $\widehat{\theta}_{N}$, makes $P_{N}=\left(\Phi_{N}^{T} \Phi_{N}\right)^{-1}$ for convenience, then eq. (5) following is held by eq. (4)

$$
\widehat{\theta}_{N}=P_{N} \Phi_{N}^{T} \mathrm{Y}_{N}
$$

Where $\widehat{\theta}_{N}$ is initial value of model parameter, then puts the next sampling time $(N+1)$ I/O data into former measurement matrix to build the output formula as following equation.

$$
\left[\begin{array}{c}
\mathrm{Y}_{N} \\
\cdots \cdots \\
y_{N+1}
\end{array}\right]=\left[\begin{array}{c}
\Phi_{N} \\
\cdots \cdots \\
\psi_{N+1}^{T}
\end{array}\right] \theta_{N+1}+\left[\begin{array}{c}
\xi_{N} \\
\cdots \cdots \\
\xi_{N+1}
\end{array}\right]
$$

Step two. According to the new output formula, basic least-squares method should be used again to acquire first recursive value $\widehat{\theta}_{N+1}$ in $(N+1)$ th sampling time. It will be more exactly all because of the newly I/O measurement data.

Step three. New brought measurement partly matrix $\psi_{N+1}$ not only increases the dimension of system measurement formula, but also amplifies the influence of measurement noise upon model parameters, so brings in a kind of recursive operator to make sure the convergence of estimation is a must. Put the recursive operator $P_{N+1}=\left(P_{N}^{-1}+\psi_{N+1} \psi_{N+1}^{T}\right)^{-1}$ into $\widehat{\theta}_{N+1}$ formula, a new recursive formula of $\hat{\theta}_{N+1}$ is given as follow.

$$
\widehat{\theta}_{N+1}=\widehat{\theta}_{N}+P_{N+1} \Psi_{N+1}\left(1+\Psi_{N+1}^{T} P_{N+1} \Psi_{N+1}\right)^{-1}\left(y_{N+1}-\Psi_{N+1}^{T} \widehat{\theta}_{N}\right)
$$

Step four. Facing the coming $(N+2)$ th sampling time I/O data $u_{N+2} 、 y_{N+2}$, recursive algorithm repeats from Step one to Step three to realize recursive least-squares system identification that also be seen as gyro random error on-line modeling.

\section{Simulation Results}

In this section, some results come from the simulated model of random error based on certain MEMS gyro's actual output in lab. Settled the angular rate is 400dps, gyro's output need to be pre-processed as following figures.
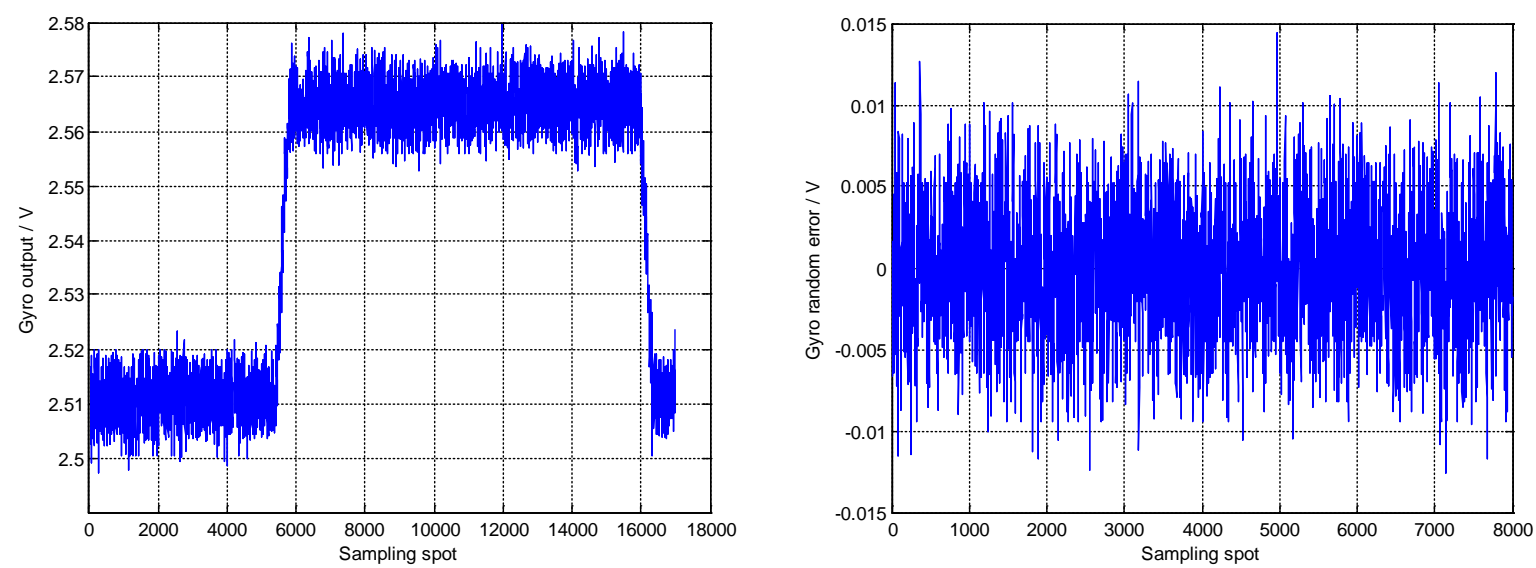

Figure 2. Gyro’s Output

Figure 3. Gyro Random Error after Pre-processing

After pre-processing, stationary testing is needed. Firstly, random sequence $x_{t}$ is cut into $M$ subsequences with equal length, then inverse number is counted and statistic $U$ is calculated, Table 1 is held by confidence coefficient $\alpha=0.05$. 
Table 1. Stationary Testing

\begin{tabular}{|c|c|c|c|c|}
\hline Confidence coef $\alpha$ & Subsequence num $M$ & Inverse num $A$ & Statistic $U$ & $U_{0}$ \\
\hline 0.05 & 10 & 26 & 0.7155 & 1.6449 \\
\hline
\end{tabular}

From table 1 , the testing result is $|\mathrm{U}|=1.6449<\mathrm{U}_{0}$, random sequence $x_{t}$ is stationary.

Secondly are periodicity testing and normality testing, toolbox of MATLAB software is required. Figure 4 shows that Power Spectral Density (PSD) of random sequence is monotone decreasing, which means $x_{t}$ is not clearly periodicity. In figure 5, normality of gyro random error sequence is proved.

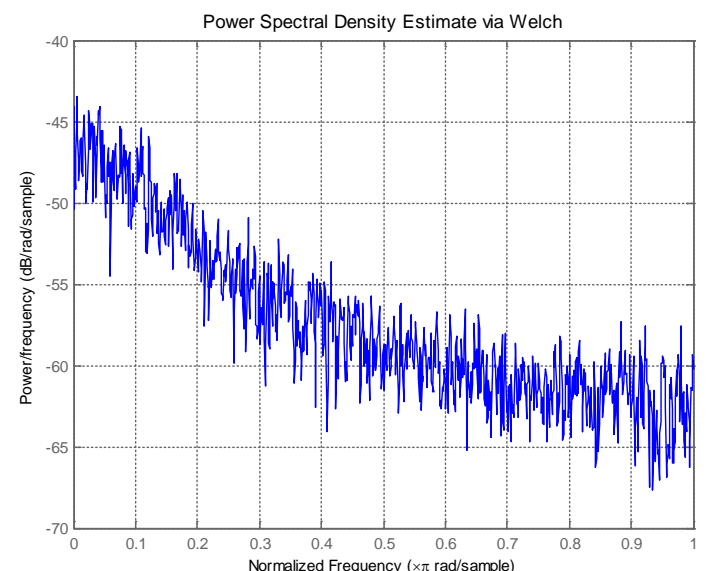

Figure 4. Power Spectral Density Curve

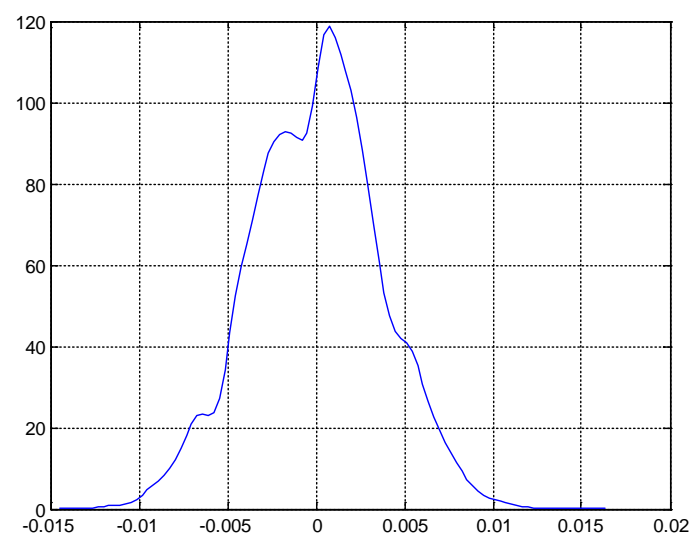

Figure 5. Normality Curve

Basing on recursive least-squares algorithm, ARMA model could be estimated on-line and the two initial values are $\hat{\theta}_{0}=\left[\begin{array}{llllll}1 & 1 & 1 & 1 & 1 & 1\end{array}\right] 、 P_{0}=1000$, then according to the AIC criteria system identified parameters are given below.

Table 2. System Parameters Identification and Oder Determination

\begin{tabular}{|c|c|c|c|c|c|c|c|}
\hline Model Order & $\phi_{1}$ & $\phi_{2}$ & $\phi_{3}$ & $\theta_{0}$ & $\theta_{1}$ & $\theta_{2}$ & AIC \\
\hline ARMA(1,1) & 0.7216 & & & 0.0065 & 0.0008 & & -9.5 \\
\hline ARMA(1,2) & 0.7364 & & & 0.0062 & 0.0005 & -0.0010 & -9.47 \\
\hline ARMA(2,1) & 0.7364 & 0.0205 & & 0.0062 & 0.0005 & & -9.44 \\
\hline
\end{tabular}

According to AIC criteria, model order ARMA $(1,1)$ is chosen and gyro random error model is shown as eq. (8).

$$
\widehat{x}_{k}=-0.7216 x_{k-1}+0.0065 u_{k}+0.0008 u_{k-1}
$$

The test of model accuracy is whether residual error between model output and gyro output approximate to white noise, the more closer means model is more accurate. Results are shown in table. 3.

Table 3. Testing Results

\begin{tabular}{|c|c|c|}
\hline Confidence Coef $\alpha$ & Statistic $U$ & $U_{0}$ \\
\hline 0.05 & 1.7992 & 5.9915 \\
\hline
\end{tabular}

From table 3, the testing result is $|\mathrm{U}|=1.7992<\mathrm{U}_{0}$, which means hypothesis of model output is approximate to white noise is accepted. In another word, this result indicates that the random error model accurately describes statistical features of gyro random error and the recursive algorithm realizes on-line modeling. Result Comparing between model output and gyro actual output is given as follow. 

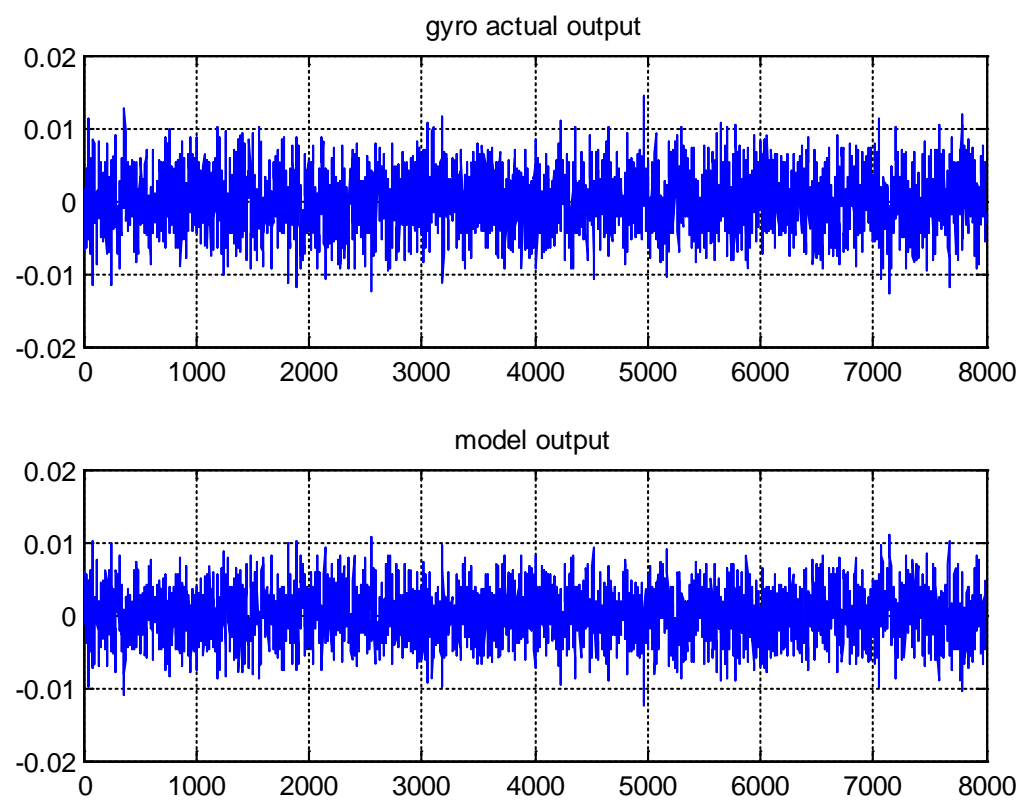

Figure 6. Gyro Actual Output and Model Output

\section{Conclusion}

This paper proposes a recursive least-squares system identification method based on time-series model to deal with the problem of gyro random error on-line modeling. With this recursive least-squares method, a few known gyro output data are used to roughly estimate system parameters, then newly obtained data are added to the measurement formula using the recursive algorithm in order to make estimated parameters approach to its truth-value. This study indicates that the residual error between outputs of gyro random error model and outputs of real gyro highly approximates white noise, which means the error model accurately describes statistical features of gyro random error and the recursive algorithm realizes on-line modeling.

\section{Acknowledgments}

This study is accomplished with a fundamental research project of State Administration of Science, Technology and Industry for National Defence (SASTIND). The authors wish to express sincere gratitude to Northwestern Polytechnical University and Science and Technology on Electromechanical Dynamic Control Laboratory.

\section{References}

[1] Sitomer J L, Kourepenis A, Connelly J H. Micromechanical Inertial Guidance Navigation and Control Systems in Gun Launched Projectiles[C]. AIAA Guidance, Navigation and Control Conference and Exhibit, Porland, 1999.

[2] R W J. Smart Munitions Development Relies Heavily on MEMS Technology[J]. Military \& Aerospace Electroniees, 2003, 14(1): 12-18.

[3] Marselli C, Amann H P, Pellandini F, et al. Error Modelling of a Silicon Angular Rate Sensor[C]. Symposium Gyro Technology, Stuttgart, Germany, 1997.

[4] Yang Peipei, Li Qing. Kalman Filtering of MEMS Gyro Based on Time-Series Model[C]. Electronic Measurement \& Instruments, ICEMI 09.9 ${ }^{\text {th }}$. International Conference. 2009, 2:367-370.

[5] Xu Kai. MEMS Gyroscopes Error Compensition Algorithm Study[J].Shenyang Ligong University, 2012 
[6] Niu Chunfeng. Gyro/GPS Integrated Attitude Measurement Method Based on Trajectory Environment[D]. Nanjing University of Science\&Technology, 2012

[7] Wang Xiufeng, Lu Guizhang. System Simulation and Identification[M]. Electronic Industary Press. 2004.7 